\title{
Dimensionality matters in the collective behaviour of active emulsions ${ }^{\star}$
}

\author{
Carsten Krüger ${ }^{\mathrm{a}}$, Christian Bahr, Stephan Herminghaus, and Corinna C. Maass \\ Max Planck Institute for Dynamics and Self-Organization (MPIDS), Am Faßberg 17, 37077 Göttingen, Germany
}

Received 22 March 2016 and Received in final form 1 June 2016

Published online: 27 June 2016

(c) The Author(s) 2016. This article is published with open access at Springerlink.com

\begin{abstract}
The behaviour of artificial microswimmers consisting of droplets of a mesogenic oil immersed in an aqueous surfactant solution depends qualitatively on the conditions of dimensional confinement; ranging from only transient aggregates in Hele-Shaw geometries to hexagonally packed, convection-driven clusters when sedimenting in an unconfined reservoir. We study the effects of varying the swimmer velocity, the height of the reservoir, and the buoyancy of the droplet swimmers. Two simple adjustments of the experimental setting lead to a suppression of clustering: either a decrease of the reservoir height below a certain value, or a match of the densities of droplets and surrounding phase, showing that the convection is the key mechanism for the clustering behaviour.
\end{abstract}

\section{Introduction}

Artificial self-propelled microswimmers have recently gained attention as model systems for mimicking biological equivalents, both for single swimmers or collective ensembles. There is particular demand for extensive ensembles of simple uniform swimmers, for example in modelling marine plankton, which forms a large part of our ecosystem $[1,2]$. A well known problem in this field is the "paradox of the plankton", i.e., the apparent conflict between the rich biodiversity in marine phytoplankton and standard predator-prey dynamics [3-5], which has been approached in studies employing chaotic oscillations [6], turbulent dynamics [7], and non-equilibrium environments in general [8].

On a less global scale, suspensions of upswimming organisms (e.g. Tetrahymena pyriformis) show collective convection involving large numbers of individual swimmers $[9,10]$. The occurrence and wavelength of these patterns depend on parameters like the reservoir height and the number density of the swimmers.

To match the complexity of biological equivalents, we need both artificial experimental and numerical systems sensitive to the interplay between self-propulsion, buoyancy, and advection. Numerical studies are highly challenging in this respect, due to the large numbers of swim-

\footnotetext{
* Contribution to the Topical Issue "Nonequilibrium Collective Dynamics in Condensed and Biological Matter", edited by Holger Stark, Marcus Baer, Carsten Beta, Sabine Klapp and Andreas Knorr.

${ }^{a}$ e-mail: carsten.krueger@ds.mpg.de
}

mers required and the resulting multi-particle dynamics, varying boundary conditions and diverse geometries, complexity of swimmer geometries and propulsion mechanisms, and the strong influence of hydrodynamic interactions. Because hydrodynamic flow fields only decay algebraically, with $1 / r$ as the leading term both in rigid spheres and droplets [11], a reliable cut-off criterion, by which the range of mutual interactions can be limited, is nearly impossible to choose. Accordingly, numerical and analytical simulations report mostly on two-dimensional (2D) systems: either rigorous $2 \mathrm{D}$ geometries [12-15], or the change in rotational diffusion properties in pure Brownian particles with varying degrees of freedom [16], or $z$ confined quasi-2D disks or rods with coupled hydrodynamic interactions [17-21,14], or swimmers confined to two dimensions interacting by three-dimensional hydrodynamic fields [22]. In the case of full three-dimensional (3D) systems, numerical simulations either exclude hydrodynamic interactions $[23,24]$ or are restricted to small system sizes [25].

In experimental studies, various artificial systems have been reported on, each showing interesting individual features. The systems can be categorized either as interface bound surfers [26-29], micro-machines [30-32], Janus particles [33-37] or active emulsions [38-47].

However, when looking for a system with high symmetry, scalability, long lifetimes, and the option to tune speed and dimensionality, most of these systems are not applicable. Either the restriction to quasi-2D geometries is inherent to the propulsion mechanism, as in the case of surfers, or the high and non-uniform mass density of the swimmers makes them ineligible for buoyancy match- 
ing, as in the case of Janus particles. For most of the active emulsion systems, the active periods are short, the chemical reactions involved are complex and the dynamics of the reaction products affect the droplet propulsion in an unpredictable manner [38-40,42-44]. Nevertheless, in certain active emulsions $[45,46]$ the propulsion mechanism is based on a solubilisation process, where the kinetics of the reaction products are diffusive and therefore analytically tractable. Due to this tractability and their high spatial symmetry, solubilising droplet swimmers appear to be well suited for three-dimensional collective studies bridging numerical and biological equivalents, if the mass density of the constituents can be buoyancy matched.

We study artificial microswimmers consisting of mesogenic oil (liquid crystal) droplets immersed in an aqueous surfactant solution [45]. The formulation is exceptionally simple (only three components - water, oil, and surfactant - are needed) and large quantities of identical swimmers can be generated in microfluidic devices. The droplets self-propel for up to one hour; the swimming speed increases with the surfactant concentration. The density of the aqueous phase can be adjusted to give the swimmers positive, neutral or negative buoyancy, and swimming dynamics can be observed in customized microfluidic geometries. Therefore, the system can be adapted to study microswimmer systems at various scales in conditions varying from single and multiple swimmers in two-dimensional confinement to full collective dynamics in three dimensions. In the experiments on high swimmer densities in variable microfluidic geometries presented below, we observed a wide range of phenomena caused by the interplay between dimensional confinement and hydrodynamic interactions. In particular, we describe a novel kind of self-assembling clustering driven by convection, which cannot be understood in a framework lacking either hydrodynamics or a full 3D description.

\section{Materials and methods}

The swimmers used in our experiments consist of droplets of the mesogenic compound 4-pentyl-4'-cyanobiphenyl (5CB) immersed into an aqueous solution of the ionic surfactant tetradecyltrimethylammonium bromide (TTAB). When the surfactant concentration $c_{s}$ exceeds the value of the critical micelle concentration $(\mathrm{CMC}=0.13 \mathrm{wt} \%)$, the droplets solubilise gradually into a homogeneous micellar nanoemulsion [48]; in which case the droplet diameter decreases linearly with time. If $c_{s}$ exceeds a threshold value of about $5 \mathrm{wt} \%$, the solubilisation is accompanied by a selfpropelled motion with velocities between 5 and $25 \mu \mathrm{m} / \mathrm{s}$, depending on the value of $c_{s}$ [45]. This can be understood in a simplified model of advection dynamics: Empty micelles advect at the front of the droplet while it dissolves, take up liquid crystal molecules and cause a gradient in surfactant coverage between the leading and the trailing end of the droplet. This corresponds to stresses leading to a self-sustained Marangoni flow in the interface. The moving interface couples to both adjacent media, causing the droplet to move with respect to the continuous phase,

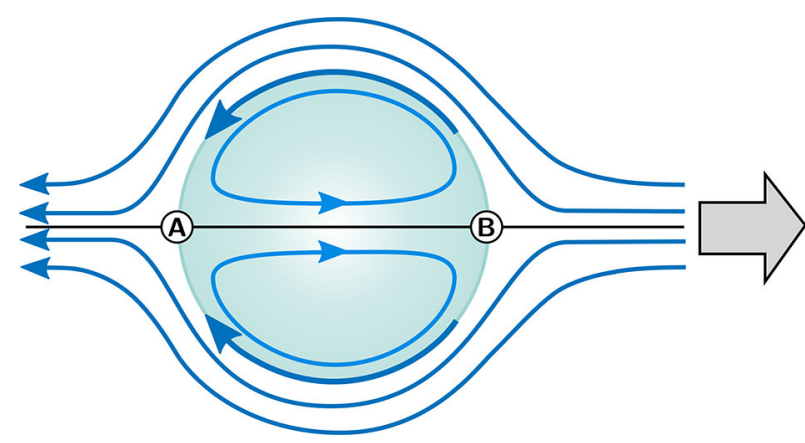

Fig. 1. Schematic of a self-propelling droplet. Blue arrows indicate the Marangoni surface flow generating internal convection and self-propelled motion. The grey arrow indicates the swimming direction. A and B are stagnation points.

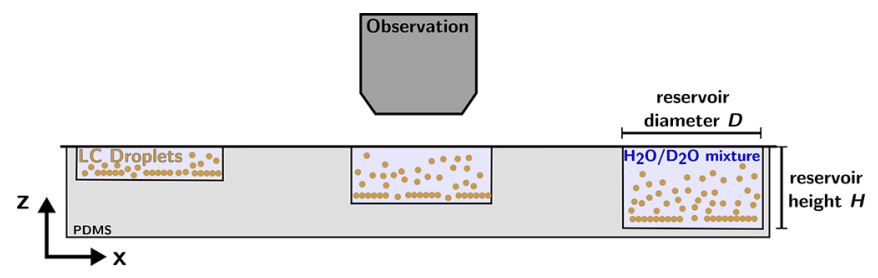

Fig. 2. Schematic of an experimental chamber. Reservoirs of different height are made by mold casting PDMS. The surfactant solution mixed with droplets is filled into the reservoirs and then closed by a cover slip, preventing evaporation. Depending on the $\mathrm{H}_{2} \mathrm{O} / \mathrm{D}_{2} \mathrm{O}$ ratio, clusters will form at the lower or upper cell boundary. The clustering is observed under bright field microscopy.

as well as convection inside the droplet (fig. 1). A more detailed description of the swimming mechanism can be found in [45].

Both 5CB (Synthon Chemicals) and TTAB (Sigma Aldrich) are used without further purifications. Droplets are mass produced in a PDMS based microfluidic flow focusing device using a surfactant solution with a concentration below the CMC, sufficiently high to stabilise the emulsion against coalescence, but low enough to keep the droplets from solubilising. The initial diameter of the droplets in the experiments presented below is $a=45 \mu \mathrm{m}$ with a polydispersity of less than $5 \%$; other sizes are available. The droplets are stored in a glass vial at densities of $\approx 1000$ droplets per $\mu \mathrm{L}$. For experiments, we use circular PDMS reservoirs with a diameter $D$ of $6 \mathrm{~mm}$ and heights $H$ varying between $0.3 \mathrm{~mm}$ and $5 \mathrm{~mm}$ (fig. 2). A small amount of the stock solution is diluted with surfactant solution at the desired concentration (between $7.5 \mathrm{wt} \%$ and $17.5 \mathrm{wt} \%$ ) and pipetted into the reservoir which is then capped with a cover slip to prevent the sample from evaporating and obscuring the field of view by forming a meniscus. We observe the in-plane droplet motion with an Olympus IX-73 bright field microscope at $2 \times$ magnification, equipped with a Canon EOS 600d camera recording data at 4 fps. For height measurements of clusters we use 


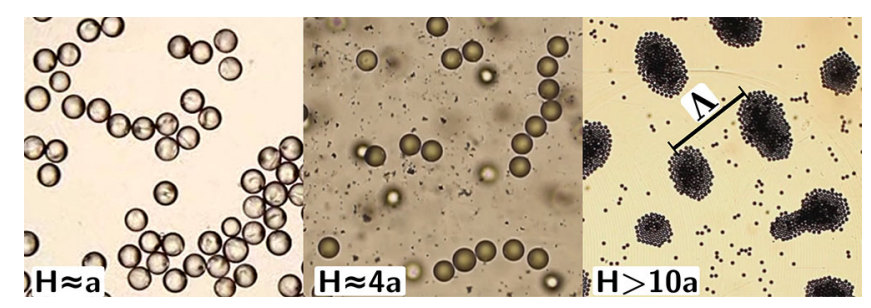

Fig. 3. The collective droplet behaviour is controlled by dimensional confinement, i.e., different ratios of reservoir height $H$ and droplet diameter $a$. In quasi-2D confinement $(H \approx a)$, there are only transient aggregates, which develop and break apart dynamically. For intermediate reservoir heights $(H \approx$ $4 a)$, hydrodynamically stabilized lines form perpendicular to the swimming direction. For unconfined reservoirs $(H>10 a)$, stable clusters with the average distance $\Lambda(H)$ assemble over large length scales. The droplet size is $a \approx 45 \mu \mathrm{m}$ in all three images. The field of view is $500 \times 500 \mu \mathrm{m}^{2}$ for left and middle, and $2.5 \times 2.5 \mathrm{~mm}^{2}$ for the right image.

the calibrated $z$-drive of the stage of an Olympus IX-83 microscope at $40 \times$ magnification. We use the same microscope and settings to map fluid motion in the aqueous phase by particle tracking or particle image velocimetry (PTV/PIV), using $2 \mu \mathrm{m}$ latex colloids as tracer particles.

There is only a small mass density difference between the liquid crystal $\left(\rho_{5 \mathrm{CB}}=1.022 \mathrm{~g} / \mathrm{cm}^{3}\right)$ and water $\left(\rho_{\mathrm{H}_{2} \mathrm{O}}=0.998 \mathrm{~g} / \mathrm{cm}^{3}\right)$. We can change the density of the surfactant solution by substituting water with heavy water $\left(\rho_{\mathrm{D}_{2} \mathrm{O}}=1.107 \mathrm{~g} / \mathrm{cm}^{3}\right)$ without affecting the chemical composition of the system. Depending on the $\mathrm{D}_{2} \mathrm{O}$ fraction the droplets either sediment, float to the top, or are neutrally buoyant.

\section{Experimental results}

\subsection{Qualitative observations}

We studied dense systems of sedimenting swimmers with initial area densities above $10 \%$ in circular reservoirs of varying height (fig. 3 ). We chose the $x y$ plane of observation to align with the reservoir bottom and the $z$ axis to point upwards against gravity (fig. 2).

When large numbers of droplets are confined to a quasi-2D layer, clustering behaviour is qualitatively similar to the low density limit of MPCD simulations by Zöttl et al. [19], showing only transient clusters (fig. 3 left). The droplets are arrested after head-on collisions until rotational diffusion enables them to leave such a transient cluster (cf. also experiments from [35]). We were not able to compare our experiments with simulations at higher number densities, because the rapid depletion of the surfactant will stop droplet motion after less than 5 minutes. Long range hydrodynamic attraction is screened by the $2 \mathrm{D}$ confinement, i.e., the short distance between swimmer and cell boundary also sets a cut-off length for the inter-particle interaction [49]. Consequently, if the reservoir height is increased to a few droplet diameters, the
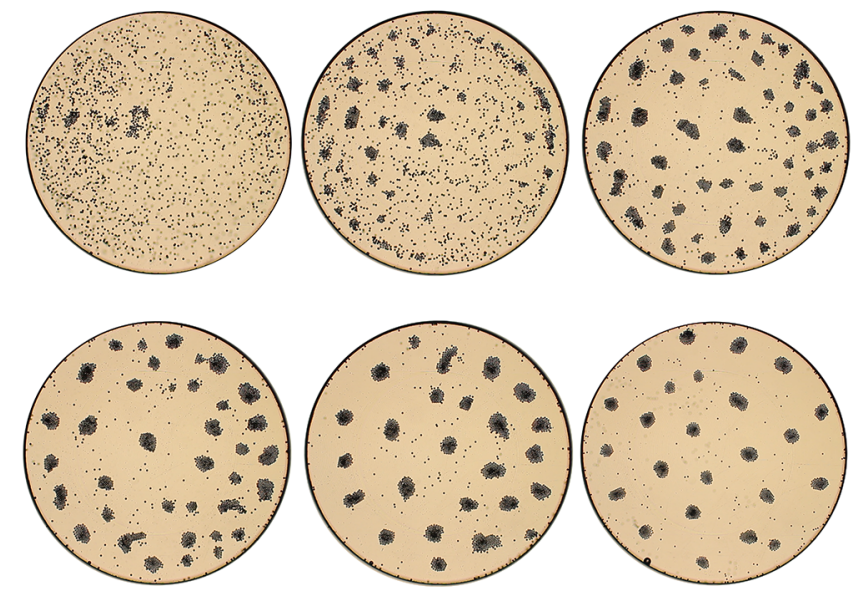

Fig. 4. Time lapse of one experiment over the course of $30 \mathrm{~min}$. The height of the reservoir is $2 \mathrm{~mm}$, the reservoir diameter is $6 \mathrm{~mm}$, the initial particle diameter is $45 \mu \mathrm{m}$, and the surfactant concentration $c_{s}$ is $10 \mathrm{wt} \%$. Top row from left to right: 0,1 and $3 \mathrm{~min}$. Bottom row from left to right: 5, 10 and $30 \mathrm{~min}$.

droplet behaviour changes (fig. 3 middle). Due to hydrodynamic attraction between the particles in the equatorial plane, droplets arrange in stable lines perpendicular to the direction of motion $[50,41,51]$ and continue collectively. These line arrangements are stable until they collide with single droplets or other lines. In a third geometric configuration extending the reservoir height to more than 7-10 droplet diameters, we observe the formation of large stable clusters of hexagonally packed monolayers (fig. 3 right), or, at higher densities, an extension into second layer packings. In the remainder of the present study, we show that this cluster formation is driven by convection and investigate the effects of surfactant concentration, reservoir height, and buoyancy adjustment.

First, we present a short summary of the life and death of a clustering swimmer system. The time series in fig. 4 shows the dynamics of cluster assembly over the course of $30 \mathrm{~min}$. A mixture of surfactant solution and individual droplets at the desired number density is injected into the reservoir. As the mass density of the swimmers exceeds that of the surfactant solution by $2 \%$, they sediment and accumulate close to the bottom of the reservoir. When the number density of the droplets near the bottom is sufficient, they nucleate into flat hexagonally packed clusters. These clusters are stable for most of the life time of the droplets (neglecting smaller rearrangements at the cluster edges) and coexist with a dilute gas-like phase of freely swimming droplets around them. The clusters hover at a finite height above the reservoir bottom and are rather stationary compared to the single swimmers. The stable regime ends when the diameter of the droplets shrinks below a certain value during dissolution. Then clusters start to disintegrate, sometimes buckling in the $z$ direction, accelerating in oblique directions and colliding with the reservoir boundary, or disassembling radially. We will confine our quantitative analysis to the stable regime, where cluster mobility is low and rearrangements are infrequent. 


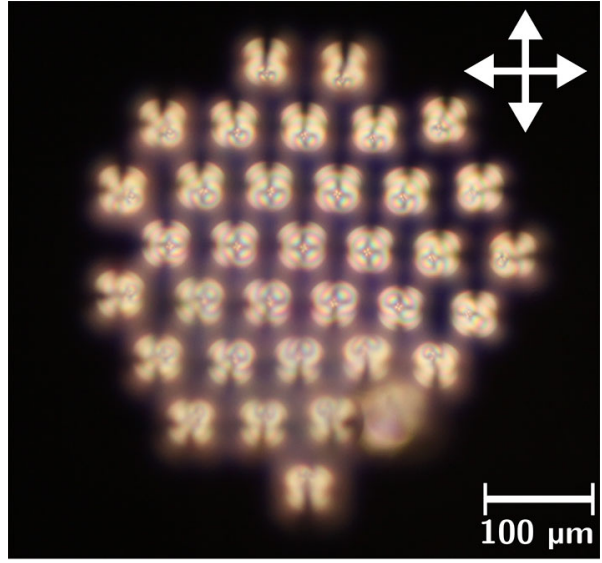

Fig. 5. Morphology of a stable cluster observed between crossed polarisers. The cross-shaped pattern inside the droplet marks the position of the defect, identifying droplets pointing upwards in the centre and tilted alignment on the boundaries with an angle to the $z$ axis of $\alpha=25 \pm 6^{\circ}$. The white double arrows show the orientation of the polariser and analyser.

\subsection{Morphology of a single cluster}

To investigate the morphology of individual clusters we make use of the optical anisotropy of the mesogenic droplet swimmers. The compound $5 \mathrm{CB}$ is nematic at room temperature, i.e. birefringent, and characteristic topological features can be observed between crossed polarisers. The coverage of the droplets with surfactant molecules leads to a perpendicular anchoring of the nematic director on the droplet surface, causing a topological point defect in the director field. While in a resting droplet the point defect is located at the centre, the internal convection in a self-propelling droplet moves the defect towards the leading edge near the flow stagnation point marked by $\mathrm{B}$ in fig. 1. Because the deformation of the director field near the point defect results in a typical pattern under a polarizing microscope, we can determine the position of the defect and the orientation of the symmetry axis of the swimming droplet. Figure 5 shows the top view on a cluster between crossed polarisers: the cross-shaped pattern inside the droplet marks the position of the defect and thus of the leading edge of the droplet. In the centre of the cluster droplets point upwards in $z$, while droplets on the boundary tilt inwards with an average angle $\alpha$ of $25 \pm 6^{\circ}$ with respect to the $z$ axis. As the droplets are oriented upwards, they pump liquid downwards, thereby generating a stagnation pressure that equilibrates the cluster at a finite height $h$ above the reservoir bottom at $z=0$. This mechanism is confirmed by the observation that $h$ increases linearly with the typical swimming velocity of a droplet swimming freely in a surfactant solution of comparable concentration (see fig. 11), i.e., $h$ increases with the pumping power of the cluster. Outflow from below the cluster and influx from above suggest a closed toroidal convection roll as sketched in fig. 6 .

For a qualitative measurement of the structure of the convection roll, we mixed the surfactant solution with $2 \mu \mathrm{m}$ latex tracer particles at a density below the single scattering limit and analysed their motion using PTV.

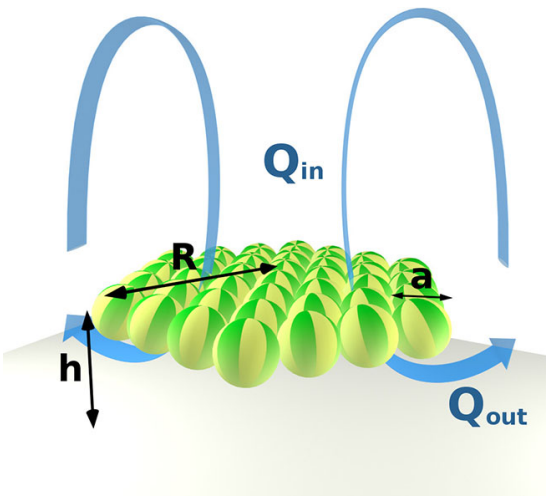

Fig. 6. Schematic of a stable cluster consisting of a single discoidal droplet layer with radius $R$ and hovering height $h$. The droplet diameter is $a$ and the stripe pattern symbolises the defect position and orientation. The volume flowing inward $Q_{\text {in }}$ equals the outward flowing volume $Q_{\text {out }}$, feeding a toroidal convection roll.

In cylindrical coordinates, we consider the centre of a cluster of radius $R$ to be located at $r=0$ and $z=h$. We determined the radial component of the flow velocity, $v_{r}$, in three sectors at different distances from the cluster edge by recording the trajectories of the tracer particles. This measurement was repeated in several focal planes in the range between $z=0$ and $z \approx 3 h$. The complete data set maps the three-dimensional profile for $v_{r}$ (fig. 7). Below the cluster, $v_{r}$ is directed along $+r$, increasing in magnitude from the reservoir bottom to a height in the middle between bottom and cluster $\left(z \approx \frac{1}{2} h\right)$ and decreasing again until the height of the cluster is reached $(z=h)$. On top of the cluster the aqueous phase is pumped inwards. The flow is directed along $-r$, with the speed increasing again up to a height $z \approx 2 h$, above which it decreases. Below the cluster, the average $v_{r}$ is larger than above the cluster. We were not able to quantitatively map tracer motion in $z$, but a flux in the direction of $+z$ for $r>R$ and $-z$ for $r<R$ exceeding the sedimentation speed of the colloids could be observed qualitatively, watching tracers move through the focal plane directly above the cluster itself, passing through the interstices between the droplets. This completes the picture of a toroidal convection roll as sketched in fig. 6 .

We can now derive a correlation between the cluster radius $R$ and the hovering height $h$ using the simple argument that the total inward flux, which enters the cluster from above, has to equal the outgoing flux, which leaves the region below the cluster in the outward direction. We consider a single-layered discoidal cluster, the centre of which is located at $r=0$ and $z=h$. Inside a cluster, droplets with radius $\frac{a}{2}$ arrange hexagonally with their leading edge pointing upwards. If a freely floating droplet moves at a speed $v_{\text {in }}$, each droplet arrested in the cluster will displace fluid at a speed $v_{\text {in }}$ times its cross section $\pi\left(\frac{a}{2}\right)^{2}$. Summation over the entire cluster, i.e., the cross section of all droplets, gives for the cluster area $A_{\mathrm{cl}}=\frac{\pi^{2}}{2 \sqrt{3}} R^{2}$ and the total inward flux $Q_{\mathrm{in}}=v_{\mathrm{in}} A_{\mathrm{cl}}$. 


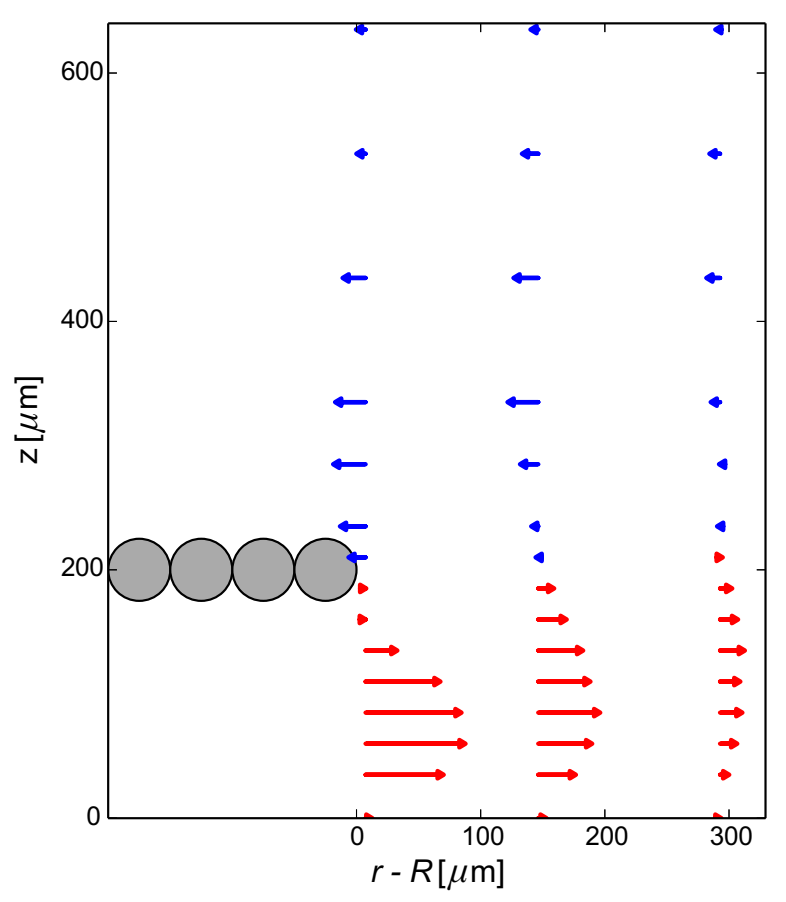

Fig. 7. Flow field around a stable cluster traced with $2 \mu \mathrm{m}$ latex particles. The grey circles show a cross section of the cluster. The radial flow velocity $v_{r}$ is determined at three different distances to the cluster edge and in various planes possessing different heights $z$ above the bottom of the reservoir $(z=0): v_{r}$ shows a Poisseuille-like profile, directed along $+r$ (away from the cluster centre) below the cluster and along $-r$ (towards the cluster centre) above.

This has to be balanced by an outward flux $Q_{\text {out }}$ which has to cross an area $A_{\mathrm{fl}}=2 \pi R h$, i.e., the boundary of a cylindrical volume with radius $R$ and height $h$ between the cluster and the bottom of the reservoir. With $v_{\text {out }}$ denoting the mean velocity of the outward flow, we have $Q_{\text {out }}=v_{\text {out }} A_{\mathrm{ff}}$. We require that

$$
Q_{\text {in }}=v_{\text {in }} A_{\mathrm{cl}} \stackrel{!}{=} v_{\text {out }} A_{\mathrm{fl}}=Q_{\text {out }} .
$$

If we calculate $Q_{\text {out }}$ by integrating the velocity data shown in fig. 7 , we find for $v_{\text {in }}$ indeed a value that corresponds approximately to the velocity of a single unconfined swimmer.

The swimmers located at the edge of the cluster have to withstand any flow directed along $+r$. Since these swimmers are tilted by an angle $\alpha$, we can assign to them a radial velocity component $v_{r}=-v_{\text {in }} \sin \alpha$. Assuming that these swimmers experience a flow of the surrounding fluid along $+r$ with a mean velocity $v_{\text {out }}$, a simple stability criterion for the cluster is $v_{\text {out }} \leq v_{\text {in }} \sin \alpha$. If $v_{\text {out }}$ is larger than this threshold, the swimmers at the cluster boundary would detach from the cluster. Replacing in eq. (1) $v_{\text {out }}$ by $v_{\text {in }} \sin \alpha$ and solving for $R$ results in

$$
R=\frac{4 \sqrt{3}}{\pi} h \sin \alpha \approx 2.2 h \sin \alpha .
$$

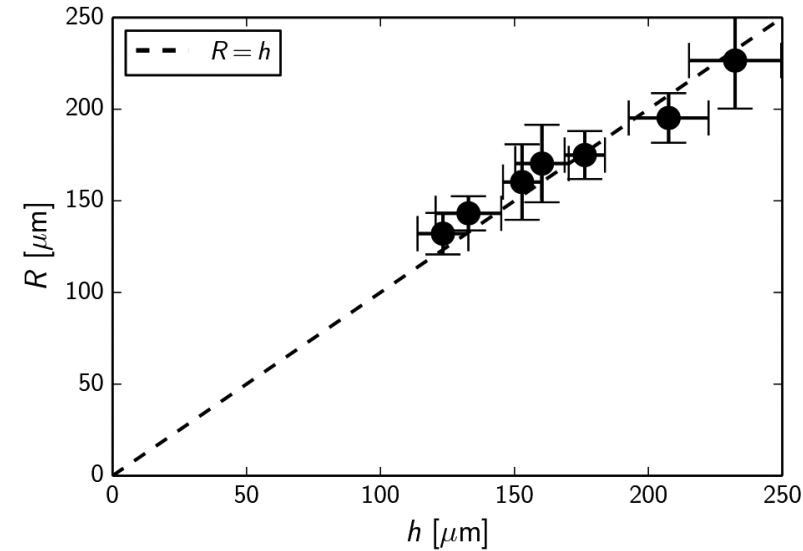

Fig. 8. Cluster radius $R$ vs. hovering height $h$. The dashed line describes the simple argument for $R(h)$ (eq. (2)) with an angle $\alpha$ of $27^{\circ}$. Chosen surfactant concentrations lay between 10 and $16 \mathrm{wt} \%$, resulting in speeds between 19 and $23 \mu \mathrm{m} / \mathrm{s}$.

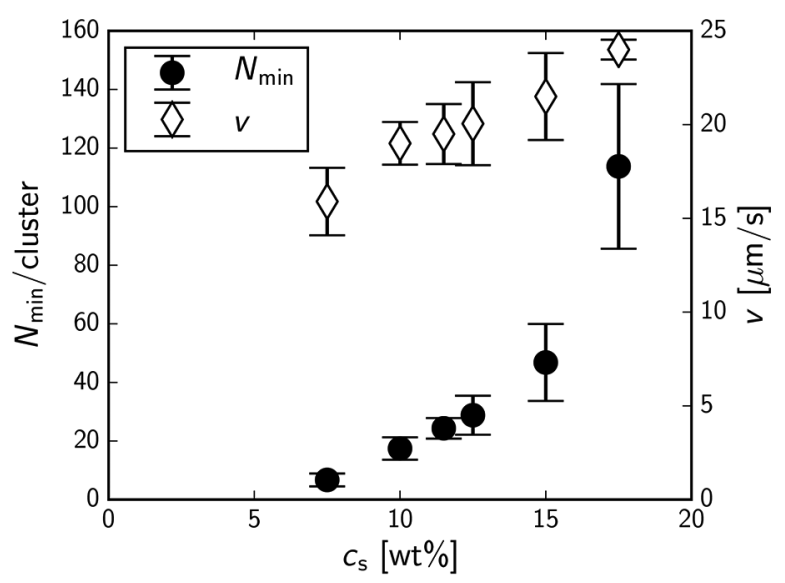

Fig. 9. Minimal droplet number for clustering, $N_{\min }$ (black circles), and droplet velocity $v$ (open diamonds) as a function of surfactant concentration $c_{s}$. The increase of velocity and decrease of reorientation time results in an increase of $N_{\min }$ at larger $c_{s}$ values.

As shown in fig. 8, we find experimentally a linear relation $R \approx h$ corresponding to $\alpha=27^{\circ}$. This is consistent with observations from polarizing microscopy (fig. 5), which suggest $\alpha=25 \pm 6^{\circ}$. An increasing droplet velocity leads to an increase of $h$ and accordingly to an increase of $R$ (see fig. 8).

The dependence of cluster formation on velocity and reorientation time in unconfined geometries can further be described by the minimum number of droplets $N_{\text {min }}$ necessary to form a cluster (see fig. 9). For low surfactant concentrations $N_{\min }$ will be smaller than for higher ones since the nucleation of a cluster depends on the speed and especially on the reorientation time of a swimmer, the first of which increases, the second decreases with surfactant concentration. For surfactants concentration as low as $7.5 \mathrm{wt} \%$, seven droplets are already enough to form a stable cluster, while for a surfactant concentration of $17.5 \mathrm{wt} \%$ more than 100 droplets are necessary to ensure 
sufficient arresting. The sharp increase of $N_{\text {min }}$ between 15 and $17.5 \mathrm{wt} \%$ suggests a threshold concentration beyond which either the pumping action of the droplets will always exceed gravity or they reorient too fast to nucleate clusters and initiate convection. Accordingly we observe no clustering when a surfactant concentration of $20 \mathrm{wt} \%$ is exceeded.

We have described how a cluster forms and which mechanism drives the observed convection roll. We will now discuss two factors influencing the large scale cluster formation, which demonstrate that the interplay between the negative buoyancy of the droplets and the hydrodynamic flow field generated by the droplets is at the heart of the mechanism for this self-assembling behaviour: first, the effect of confining the convection roll by decreasing the height of the reservoir (sect. 3.3) and second, the clustering behaviour of droplets in various buoyancy settings (sect. 3.4).

\subsection{Effect of confining the convection roll on the clustering behaviour}

As described in sect. 3.1, a certain minimum height $H_{\text {min }}$ of the reservoir is required in order to observe the formation of the convection-stabilized clusters. The value of $H_{\text {min }}$ depends on the droplet velocity $v$ : it varies from $H_{\text {min }} \approx 0.25 \mathrm{~mm}$ for $v=19 \mu \mathrm{m} / \mathrm{s}$ to $H_{\text {min }} \approx 1.5 \mathrm{~mm}$ for $v=24 \mu \mathrm{m} / \mathrm{s}$. Since the swimmer velocity determines the flow velocity in the convection roll, and thus the spatial extent of the convection roll, the observed dependence of $H_{\text {min }}$ on $v$ is a first indication of the importance of convection for the formation of the clusters: for $H<H_{\min }$, clusters do not form because there is no space for the convection roll to develop.

In a certain range of reservoir heights $H$ above $H_{\text {min }}$ we still observe a pronounced effect of $H$ on the clustering behaviour. For $H$ just above $H_{\min }$, a convection roll develops, but has to be smaller than in the unconfined case $H \gg H_{\min }$, as it is compressed between bottom and ceiling of the reservoir. A direct measure of the lateral size of the convection rolls is the average distance $\Lambda$ between neighbouring clusters (see right panel of fig. 3). If we assume a roughly circular cross section of the convection torus, we can expect an asymptotic limit of $\Lambda=2 H$ for shallow reservoirs, independent of swimmer speed. The relation is asymptotical because we cannot exclude compression due to the finite lateral confinement. We measured $\Lambda(H)$, varying $H$ stepwise by changing reservoirs, for two swimmer systems with roughly equal swimmer numbers (3500 droplets) and different free swimming speeds set by the TTAB concentration. The first system uses a $c_{s}$ of $11.5 \mathrm{wt} \%$, corresponding to a free swimming speed of $19.5 \mu \mathrm{m} / \mathrm{s}$ and a minimum $H_{\min }$ for clustering of $\approx 0.45 \mathrm{~mm}$. The corresponding values for the second system are $9 \mathrm{wt} \%, 15.5 \mu \mathrm{m} / \mathrm{s}$ and $0.35 \mathrm{~mm}$. We have plotted the dependence of $\Lambda$ on $H$ in fig. 10. For shallow reservoirs, $H_{\text {min }}<H<3 H_{\text {min }}, \Lambda=2 H$ is indeed a reasonable asymptote for both data sets. For higher reservoirs, $H$ exceeds the natural extent of the convection roll and $\Lambda(H)$ approaches a constant value that increases

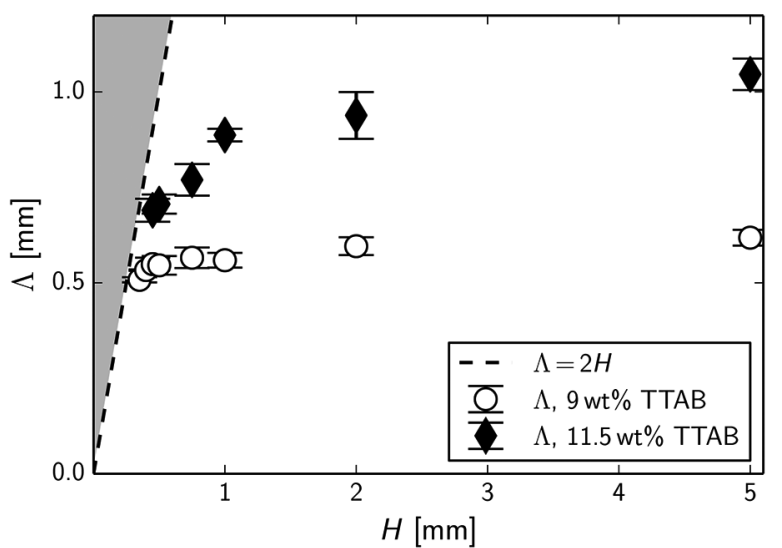

Fig. 10. Average cluster distance $\Lambda$ for different reservoir heights $H$. For shallow reservoirs, we observe a linear asymptote $\Lambda=2 H$ (dotted line), beyond which clustering is entirely suppressed (shaded area), for the transition to containers unconfined in $z$, a plateauing value. The two systems consisted of $\approx 3500$ droplets (diameter $45 \mu \mathrm{m}$ ) with surfactant concentration $c_{s}=9 \mathrm{wt} \%$ and droplet velocity $v=15.5 \mu \mathrm{m} / \mathrm{s}$ (open circles), and $c_{s}=11.5 \mathrm{wt} \%$ and $v=19.5$ (black diamonds).

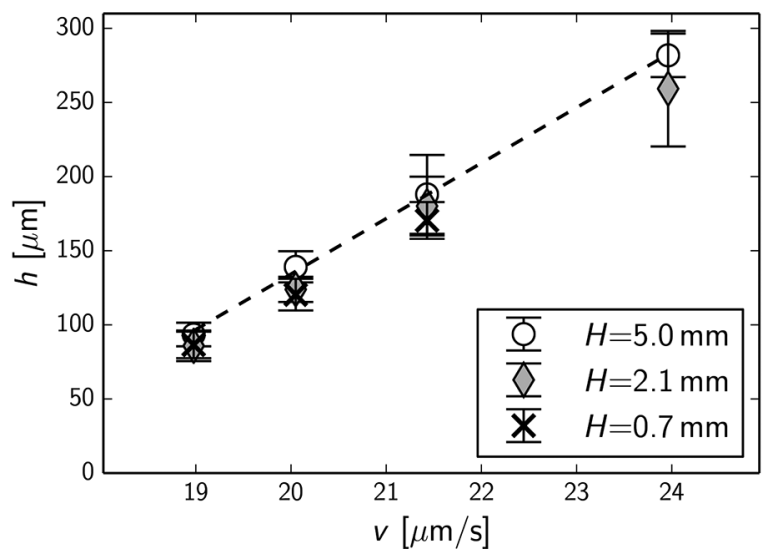

Fig. 11. Hovering height $h$ as a function of the swimmer velocity $v$. Symbols correspond to different reservoir heights $H$. The dashed line marks a linear fit for $h(v)$ for unconfined reservoirs $(H=5 \mathrm{~mm})$.

with the swimming speed, i.e. the pumping power driving the convection roll.

The reservoir height $H$ has also a subtle influence on the relation between hovering height $h$ and droplet velocity $v$. As described in sect. 3.2, the clusters hover at a certain distance $h$ above the reservoir bottom as a result of the stagnation pressure generated by the arrested droplets pumping fluid towards the bottom. Accordingly, $h$ increases linearly with the droplet velocity $v$ which determines the pumping power of the cluster. If the convection is distorted by decreasing $H$, we expect additional pressure contributions from the ceiling of the reservoir and thus a decrease of $h$. Figure 11 shows $h$ as a function of $v$ for three different reservoir heights $H$. The linear dependence of $h$ on $v$ is obviously not affected by $H$. However, for a fixed value of $v$ we observe a small decrease of $h$ when 


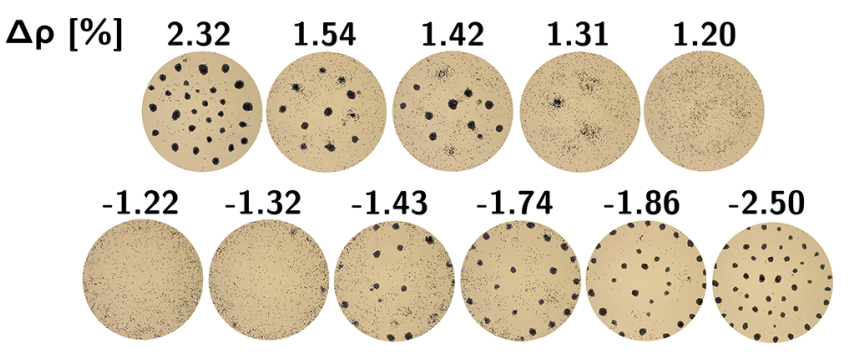

Fig. 12. Buoyancy adjustment and cluster formation in a sample with $c_{s}=10 \mathrm{wt} \%$. At a critical density difference $\Delta \rho_{+}$the formation of clusters ceases and appears again for negative density differences at a threshold $\Delta \rho_{-}$. The reservoir height is $2 \mathrm{~mm}$ and the initial droplet diameter is $45 \mu \mathrm{m}$. Images are taken 20 min after injecting the droplets.

$H$ is decreased from $5 \mathrm{~mm}$ to $0.7 \mathrm{~mm}$, indicating again the confinement of the convection roll by the reservoir geometry.

\subsection{Effect of varying the buoyancy on the clustering behaviour}

Finally, we report on the effect of varying the buoyancy of the droplet swimmers on their clustering behaviour. The density of $5 \mathrm{CB}, \rho_{5 \mathrm{CB}}$, is slightly larger than the density of the aqueous TTAB solution, $\rho_{\text {aq }}$, and the droplets sediment at the bottom of the reservoir. We can continuously adjust the density difference $\rho_{5 \mathrm{CB}}-\rho_{\text {aq }}$ without changing the chemical properties of our system by preparing TTAB solutions with variable $\mathrm{H}_{2} \mathrm{O} / \mathrm{D}_{2} \mathrm{O}$ ratio and constant surfactant concentration $c_{s}$. If we approach buoyancy matching while the swimmer velocity is kept constant, swimmers can easily escape a region where a cluster starts forming and the cluster-stabilizing convection cannot develop. Clustering is suppressed when the stagnation pressure below the cluster exceeds gravity.

We added a fixed quantity of 2000-2500 droplets to the aqueous phase and checked for cluster formation during a time interval of $20 \mathrm{~min}$. Figure 12 shows a sequence of images, recorded $20 \mathrm{~min}$ after the start of the experiment, for $c_{s}=10 \mathrm{wt} \%$ and decreasing values of

$$
\Delta \rho=\frac{\rho_{5 \mathrm{CB}}-\rho_{\mathrm{aq}}}{\left(\rho_{5 \mathrm{CB}}+\rho_{\mathrm{aq}}\right) / 2} .
$$

No clustering is observed for $\Delta \rho \lesssim 1.42 \%$, while clusters reappear for $\Delta \rho \lesssim-1.43 \%$. As the swimmers float in this case, the clusters for $\Delta \rho<0$ form at the ceiling of the reservoir. Figure 13 summarises the two threshold values for cluster suppression, $\Delta \rho_{+}$for sedimenting and $\Delta \rho_{-}$for floating droplets, for the different values of $c_{s}$. The absolute values of $\Delta \rho_{+}$and $\Delta \rho_{-}$are roughly equal for a given surfactant concentration or droplet velocity, indicating that clusters consisting of floating droplets are based on the same convection based mechanism as the clusters of sedimenting droplets. The slight differences in slope between the two cases (see fig. 13) could be due to the different materials forming the ceiling (glass) or bottom (PDMS) of the reservoir.

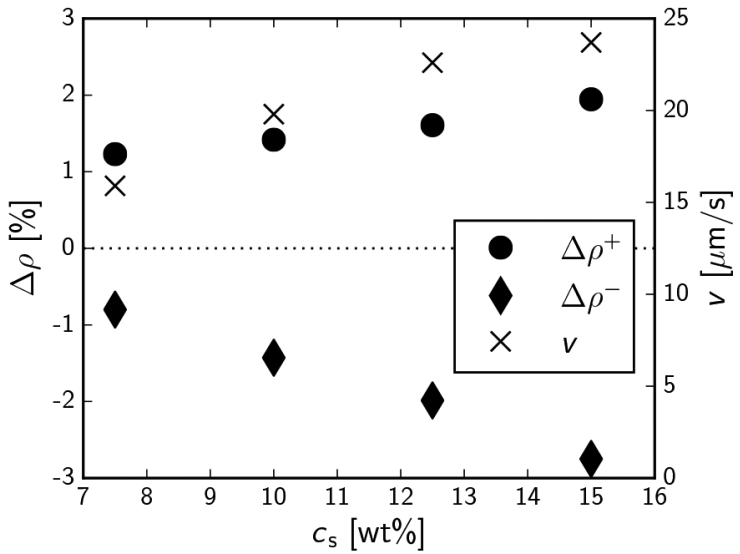

Fig. 13. Values of the clustering thresholds $\Delta \rho_{+}$(circles) and $\Delta \rho_{-}$(diamonds) for different surfactant concentrations $c_{s}$. Corresponding droplet velocities $v$ are marked by crosses.

\section{Conclusions and outlook}

We have demonstrated that a full treatment of dimensionality, geometry, and hydrodynamics is necessary to understand the collective dynamics of active swimmers, as we have observed convective patterns strongly influenced by the geometry of the system. In the case of pure geometrical frustration in active Brownian swimmers $[16,13,35]$, clustering should be suppressed by increasing dimensional degrees of freedom, which, in contrast, stresses the importance of hydrodynamic interactions in our system of more persistent swimmers. Despite the complexity of clustering features in our experiments, all these can be well explained by considering and adjusting buoyancy, geometry and hydrodynamics, without having to account for system specific parameters like swimmer morphology or chemistry. This is highly relevant to studies comparing to biological systems, as many systems in nature either create bioconvective patterns by themselves (e.g. Tetrahymena pyriformis) or exhibit traits possibly caused by interaction with external flows, e.g. the species diversity problem stated in the plankton paradox. We propose our system as a well suited candidate for comparison with complex biological systems, especially as the swimmers are perfect spheres and external forces like gravity can be tuned, which facilitates hydrodynamic modelling. The swimmers are highly scalable in numbers and sizes and both swimmer dynamics and flow fields can be easily observed and tracked by optical microscopy at all length scales relevant to the system.

Furthermore, we have employed the findings presented in the main body of this paper in a number of demonstration experiments. To show that the swimmers "sense" the geometry of the space available to them, even without lateral confinement, we investigated a geometry where swimmers float in a high $\mathrm{D}_{2} \mathrm{O}$ fraction, $\Delta \rho=-4.35 \%$, at the ceiling of a $z$-confined cell $(H=300 \mu \mathrm{m})$, the bottom of which contains circular reservoirs with depth varying between 0.5 and $5 \mathrm{~mm}$ (see drawing in fig. 14). The droplet 


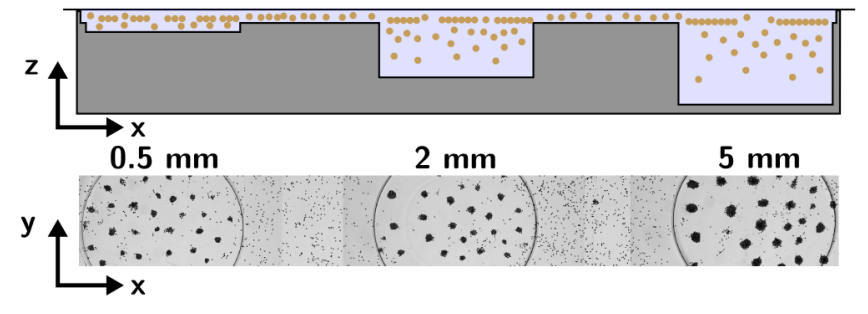

Fig. 14. Surface patterning by dimensionality in a two-layer reservoir geometry. The top layer is $300 \mu \mathrm{m}$ high, below the clustering limit. The second layer embeds reservoirs of depth between 0.5 and $5 \mathrm{~mm}$ and $6 \mathrm{~mm}$ in diameter. The surfactant solution has a concentration of $15 \mathrm{wt} \%$ and, via $\mathrm{D}_{2} \mathrm{O}$ admixture, a negative density difference $\Delta \rho=-4.35 \%$, such that droplets float at the ceiling. The droplet diameter is $45 \mu \mathrm{m}$. Top: schematic of the experimental setup. Bottom: cluster formation after $20 \mathrm{~min}$.

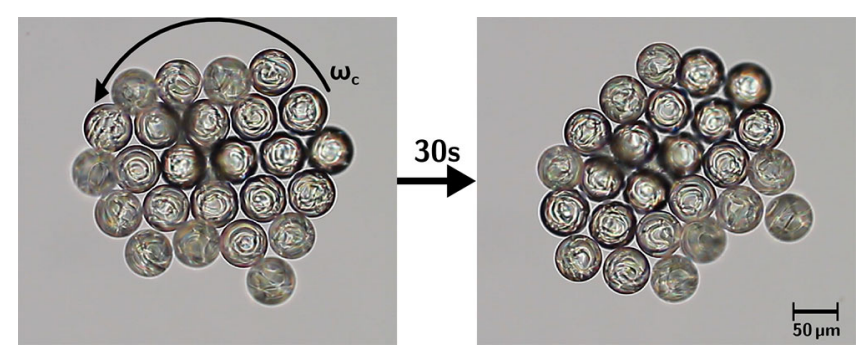

Fig. 15. Still images of a counterclockwise rotating cluster assembled out of nematic droplet swimmers containing a chiral dopant. The elapsed time between the two images is $30 \mathrm{~s}$.

swimmers were free to move across the entire area but formed clusters only above the wells at the cell bottom (lower panel of fig. 14), with cluster spacing and sizes increasing with the well depth.

We observed a second interesting feature when adding a small amount of a chiral dopant (Merck, R811/S811) to our nematic droplets. In a chiral nematic phase, the director field forms a helical superstructure. We find that nematic droplets containing a chiral dopant not only translate while moving but also rotate. In a cluster formed by chiral droplets, the rotation of the droplets adds a tangential velocity to the cluster, resulting in a rotation of the whole cluster, similar as observed for aggregates of the bacterium Thiovulum majus [52]. Figure 15 shows a micrograph of a rotating cluster consisting of chiral nematic droplet swimmers. The rotation period is in the range of $160-300 \mathrm{~s}$. The rotation of the cluster due to molecular chirality is easily confirmed by adding either R or S enantiomers of the chiral dopant: while for one enantiomer a clockwise rotation of the clusters is observed, clusters containing the enantiomer with the opposite handedness rotate counterclockwise. Non-doped clusters do not rotate. In this respect, our system differs from simulations of dumbbell aggregates $[53,54]$ and studies of bacterial micro-rotors [55], where rotation is caused by the random addition of individual torques and there is no global chirality in the system.

\section{Author contribution statement}

CK designed and performed experiments, analysed data and wrote the paper, CCM supervised the study, wrote analysis software and wrote the paper. CB wrote the paper, $\mathrm{CB}$ and $\mathrm{SH}$ discussed results and implications and edited the manuscript.

Open access funding provided by Max Planck Society (or associated institution if applicable). Financial support from the Deutsche Forschungsgemeinschaft (Priority Programme SPP1726 "Microswimmers") is gratefully acknowledged. We thank one Referee for pointing out ref. [16] to us.

Open Access This is an open access article distributed under the terms of the Creative Commons Attribution License (http://creativecommons.org/licenses/by/4.0), which permits unrestricted use, distribution, and reproduction in any medium, provided the original work is properly cited.

\section{References}

1. M. Simon, B.C. Cho, F. Azam, Mar. Ecol. Prog. Ser. 86, 103 (1992)

2. J.P. Descy, V. Gosselain, Hydrobiologia 289, 139 (1994).

3. G. Hardin, Science 131, 1292 (1960).

4. G.E. Hutchinson, Am. Nat. 95, 137 (1961).

5. U. Sommer, J. Padisak, C.S. Reynolds, P. Juhasz-Nagy, Hydrobiologia 249, 1 (1993).

6. J. Huisman, F.J. Weissing, Nature 402, 407 (1999).

7. F. D'Ovidio, S. De Monte, S. Alvain, Y. Dandonneau, M. Lévy, Proc. Natl. Acad. Sci. U.S.A. 107, 18366 (2010).

8. M. Scheffer, S. Rinaldi, J. Huisman, F.J. Weissing, Hydrobiologia 491, 9 (2003).

9. T. Pedley, J. Kessler, Annu. Rev. Fluid Mech. 24, 313 (1992).

10. N. Hill, T. Pedley, Fluid Dyn. Res. 37, 1 (2005).

11. E. Guyon, J.P. Hulin, L. Petit, C.D. Mitescu, Physical Hydrodynamics, 2nd edition (Oxford University Press, 2015).

12. H.H. Wensink, H. Löwen, J. Phys.: Condens. Matter 24, 464130 (2012).

13. J. Bialké, H. Löwen, T. Speck, EPL 103, 30008 (2013).

14. A.M. Menzel, T. Ohta, H. Löwen, Phys. Rev. E 89, 022301 (2014).

15. P. Cremer, H. Löwen, Phys. Rev. E 89, 022307 (2014).

16. B. ten Hagen, S. van Teeffelen, H. Löwen, J. Phys.: Condens. Matter 23, 194119 (2011).

17. M. Leoni, T.B. Liverpool, Phys. Rev. Lett. 105, 238102 (2010).

18. N. Desreumaux, N. Florent, E. Lauga, D. Bartolo, Eur. Phys. J. E 35, 68 (2012).

19. A. Zöttl, H. Stark, Phys. Rev. Lett. 112, 118101 (2014).

20. R. Matas-Navarro, R. Golestanian, T.B. Liverpool, S.M. Fielding, Phys. Rev. E 90, 032304 (2014).

21. M. Leoni, T. Liverpool, Phys. Rev. Lett. 112, 148104 (2014).

22. T. Ishikawa, T. Pedley, Phys. Rev. Lett. 100, 088103 (2008).

23. J. Stenhammar, D. Marenduzzo, R.J. Allen, M.E. Cates, Soft Matter 10, 1489 (2014).

24. J. Stenhammar, R. Wittkowski, D. Marenduzzo, M.E. Cates, Phys. Rev. Lett. 114, 018301 (2015). 
25. G.J. Li, A.M. Ardekani, Phys. Rev. E 90, 013010 (2014).

26. F. Dos Santos, T. Ondarçuhu, Phys. Rev. Lett. 75, 2972 (1995).

27. T. Ban, T. Kita, H. Tominaga, S. Akihisa, Chem. Lett. 37, $162(2008)$.

28. Y.J. Chen, Y. Nagamine, K. Yoshikawa, Phys. Rev. E 80 , 016303 (2009).

29. J. Palacci, S. Sacanna, A.P. Steinberg, D.J. Pine, P.M. Chaikin, Science 339, 936 (2013).

30. R. Dreyfus, J. Baudry, M.L. Roper, M. Fermigier, H.A. Stone, J. Bibette, Nature 437, 862 (2005).

31. A. Ghosh, P. Fischer, Nano Lett. 9, 2243 (2009).

32. A. Snezhko, M. Belkin, I.S. Aranson, W.K. Kwok, Phys. Rev. Lett. 102, 118103 (2009).

33. J. Howse, R. Jones, A. Ryan, T. Gough, R. Vafabakhsh, R. Golestanian, Phys. Rev. Lett. 99, 048102 (2007).

34. G. Volpe, I. Buttinoni, D. Vogt, H.J. Kümmerer, C. Bechinger, Soft Matter 7, 8810 (2011).

35. I. Buttinoni, J. Bialké, F. Kümmel, H. Löwen, C. Bechinger, T. Speck, Phys. Rev. Lett. 110, 238301 (2013).

36. B. ten Hagen, F. Kuemmel, R. Wittkowski, T. Daisuke, H. Löwen, C. Bechinger, Nat. Commun. 5, 4829 (2014).

37. F. Kuemmel, P. Shabestari, C. Lozano, G. Volpe, C. Bechinger, Soft Matter 11, 6187 (2015)

38. T. Toyota, H. Tsuha, K. Yamada, T. Katsuto, T. Ikegami, T. Sugawara, Chem. Lett. 35, 708 (2006).

39. M.M. Hanczyc, T. Toyota, T. Ikegami, P. Norman, T. Sugawara, J. Am. Chem. Soc. 129, 9386 (2007).

40. T. Toyota, N. Maru, M.M. Hanczyc, T. Ikegami, T. Sugawara, J. Am. Chem. Soc. 131, 5012 (2009).
41. S. Thutupalli, R. Seemann, S. Herminghaus, New J. Phys. 13, 73021 (2011)

42. T. Banno, R. Kuroha, T. Toyota, Langmuir 28, 1190 (2012).

43. T. Ban, T. Yamagami, H. Nakata, O. Yasunori, Langmuir 29, 2554 (2013).

44. T. Banno, S. Miura, R. Kuroha, T. Toyota, Langmuir 29, 7689 (2013)

45. S. Herminghaus, C.C. Maass, C. Krüger, S. Thutupalli, L. Goehring, C. Bahr, Soft Matter 10, 7008 (2014).

46. Z. Izri, M.N. van der Linden, S. Michelin, O. Dauchot, Phys. Rev. Lett. 113, 248302 (2014).

47. C.C. Maass, C. Krüger, S. Herminghaus, C. Bahr, Annu. Rev. Conden. Matter Phys. 7, 171 (2016).

48. K. Peddireddy, P. Kumar, S. Thutupalli, S. Herminghaus, C. Bahr, Langmuir 28, 12426 (2012).

49. J. Happel, H. Brenner, Low Reynolds Number Hydrodynamics (Prentice Hall, NJ, 1965).

50. I.O. Götze, G. Gompper, Phys. Rev. E 82, 041921 (2010).

51. N. Aguillon, A. Decoene, B. Fabrèges, B. Maury, B. Semin, ESAIM: Proc. 38, 36 (2013).

52. A.P. Petroff, X.L. Wu, A. Libchaber, Phys. Rev. Lett. 114, $158102(2015)$.

53. V.B. Putz, J. Dunkel, J.M. Yeomans, Chem. Phys. 375, $557(2010)$

54. C. Tung, J. Harder, C. Valeriani, A. Cacciuto, Soft Matter 12, 555 (2016).

55. J. Schwarz-Linek, C. Valeriani, A. Cacciuto, M.E. Cates, D. Marenduzzo, A.N. Morozov, W.C. Poon, Proc. Natl. Acad. Sci. U.S.A. 109, 4052 (2012). 